\title{
Helicobacter pylori: ¿cómo mejorar las terapias de erradicación?
}

\section{How can Helicobacter pylori eradication therapies be improved?}

\author{
Jorge Morcillo Muñoz, MD, ${ }^{1}$ William Otero Regino, MD, ${ }^{2 *}$ Martín Gómez Zuleta, MD. ${ }^{3}$
}

\footnotetext{
' Médico Cirujano, Universidad Nacional de Colombia Bogotá D. C., Colombia

2 Profesor Titular de Medicina, Unidad de

Gastroenterología, Universidad Nacional de Colombia, Hospital Universitario Nacional.

Gastroenterólogo, Clínica Fundadores. Bogotá D. C. Colombia.

3 Profesor Asociado de Medicina, Unidad de Gastroenterología, Universidad Nacional de

Colombia, Hospital Universitario Nacional.

Gastroenterólogo, Hospital de Kennedy.

Bogotá D. C., Colombia.

*Correspondencia: waoteror@gmail.com.
}

Fecha recibido: $14-02-18$

Fecha aceptado: 02-05-18

\begin{abstract}
Resumen
Desde el descubrimiento de Helicobacter pylori, la erradicación del mismo ha sido un reto constante y el tratamiento ideal todavía no está disponible. La creciente resistencia del microorganismo a los antibióticos más frecuentemente utilizados es la circunstancia que más ha impactado en la eficacia de los diferentes esquemas. El objetivo del presente trabajo es revisar los aspectos generales de la infección por $H$. pylori, los aspectos básicos de los componentes de las terapias de erradicación, la nomenclatura de los distintos tratamientos, las características generales de los antibióticos y, finalmente, los tratamientos recomendados con base en las publicaciones recientes más importantes.
\end{abstract}

\section{Palabras clave}

Helicobacter, resistencia, inhibidores de la bomba de protones, antibióticos.

\section{Abstract}

Since the discovery of Helicobacter pylori (H. pylori), eradication has been a constant challenge, and an ideal treatment is not yet available. The increasing resistance of the microorganism to the most frequently used antibiotics has most impacted the effectiveness of the various schemes. The objectives of this work are to provide a general review of $H$. pylori infections and the nomenclature of the various treatments, discuss the basic issues and components of eradication therapies together with the general characteristics of antibiotics, and finally to recommend treatments based on in the most important recent publications.

\section{Keywords}

Helicobacter, resistance, PPI, antibiotics.

\section{INTRODUCCIÓN}

Helicobacter pylori es una bacteria gramnegativa, microaerófila y capaz de colonizar el estómago (1). Produce gastritis crónica, úlceras pépticas, cáncer gástrico (CG) y enfermedades hematológicas (1-3). Representa la infección bacteriana crónica más común en el ser humano después de Streptococcus mutans, productor de las caries dentales (4); en 2015 había aproximadamente 4400 millones de personas infectadas (5). El continente más afectado es África y el menos afectado es
Oceanía, con prevalencias de $70 \%$ y $24 \%$, respectivamente (5). Nigeria tiene la prevalencia más alta $(87,7 \%)$ y Suiza, la más baja (19\%). En los países con alta incidencia de CG, la prevalencia de $H$. pylori es el doble que la de los países con baja incidencia de ese tumor $(6,7)$. La mejoría de las condiciones sanitarias y la erradicación de los infectados identificados disminuyen la prevalencia de la infección (7). La tasa de reinfección posterradicación se relaciona con el nivel socioeconómico de los diferentes países (8-10). En Japón es del $2 \%$ y en América Latina del $8 \%(8,9)$. 
El papel etiológico de H. pylori en gastritis crónica y úlceras pépticas fue demostrado por Warren y Marshall en 1984 (11). Por este hallazgo recibieron el premio Nobel de medicina en el 2005 (12). El $20 \%$ de todos los infectados desarrollará alguna enfermedad digestiva como úlceras pépticas, CG o linfoma MALT (linfoma de tejido linfoide asociado a mucosa) gástrico (13-16). En 1994 la Organización Mundial de la Salud (OMS) clasificó a H. pylori como un carcinógeno tipo I o carcinógeno definido (17). Para muchos países latinoamericanos, incluida Colombia, el CG es un problema de salud pública; y en la reciente guía colombiana de práctica clínica sobre CG, se recomienda erradicar H. pylori en todos los infectados para disminuir el riesgo de ese tumor (18). Países como Taiwán y China han demostrado que erradicar H. pylori disminuye la incidencia de CG en $25 \%$ y $39 \%$, respectivamente (19). El reciente consenso de Kioto ratificó la recomendación de erradicar $H$. pylori a todos los infectados, independientemente de la severidad de la gastritis y los síntomas (20). Pero todavía no se dispone de esquemas con eficacia superior al $98 \%$, exigida para las enfermedades infecciosas y, por el contrario, frecuentemente su éxito es $80 \%$ o menos (inaceptable) $(3,21)$.

Los frecuentes fracasos de las terapias utilizadas están relacionados con factores del microorganismo y del huésped (21-30). Los factores de H. pylori incluyen su hábitat ácido, microaerofilia, formación de biofilm (que lo protege de los antibióticos), alta carga bacteriana, con múltiples individuos durmientes (etapa no replicativa), evasión inmunológica y resistencia a los antibióticos (21-23). Este último es el factor más importante (21-25). La resistencia a la claritromicina, metronidazol (21-24) y más recientemente a quinolonas (22) ha originado terapias cada vez menos eficaces a nivel mundial $(3,4,21-27)$.

En el presente artículo se revisarán los conceptos básicos de las terapias de erradicación y los factores adicionales implicados en el éxito de los tratamientos.

\section{INHIBICIÓN PROFUNDA DE LA SECRECIÓN DE ÁCIDO}

Los inhibidores de la bomba de protones (IBP; omeprazol, esomeprazol, lansoprazol, rabeprazol, entre otros) son medicamentos fundamentales en los diferentes esquemas de tratamiento. $\mathrm{Al}$ inhibir la secreción del ácido gástrico y elevar el $\mathrm{pH}$ por encima de 6 , se aumenta la replicación de $H$. pylori y se aumenta la eficacia de los antibióticos (3, 24-30). Sin embargo, la eficacia de los IBP es influida por el metabolismo de los mismos en el citocromo $\mathrm{P} 450$ (CYP450) y del genotipo particular de CYP219C, y otros como CYP3A4, ABCB1 y niveles de IL- $1 \beta$ (24-30). De todos estos, el CYP2C19 es el más importante (24-29) y el impacto es mayor con los esquemas que utilizan omeprazol y lansoprazol, y menos en los que se incluyen esomeprazol y rabeprazol (25-27). Este último medicamento tiene un metabolismo independiente de ese sistema enzimático (2527). Con base en el polimorfismo genético del CYP2C19, los individuos se clasifican en 3 categorías de metabolizadores de los IBP: metabolizadores rápidos (MR), metabolizadores intermedios (MI) y metabolizadores lentos (ML) (2, 28-30), los cuales varían en las diferentes poblaciones (28-31). Los asiáticos tienen alta prevalencia de ML; mientras que en las poblaciones caucásicas la mayoría es MR $(29,30)$. Sin embargo, cuando el lansoprazol o el omeprazol, se dosifican 4 veces al día, hay suficiente supresión de ácido en los MR de $(24,26,27)$.

Estudios realizados en Japón hace más de 20 años demostraron que la erradicación de $H$. pylori con terapia dual (amoxicilina e IBP) varía según el CYP2C19 $(27,28)$, siendo $28 \%, 60 \%$ y $100 \%$, respectivamente en los MR, MI y ML; y de manera similar se comportó la curación de las úlceras pépticas (28). En un metaanálisis reciente (29), se encontró que las tasas de éxito terapéutico de los MR fueron inferiores a las de los MI independientemente del tipo de IBP que se tomara (28). No se observaron diferencias significativas para esomeprazol y rabeprazol (29). En Corea del Sur se ha encontrado que un factor de riesgo para falla del tratamiento de erradicación es ser MR (Odds ratio [OR]: 1,84; intervalo de confianza [IC] $95 \%: 1,04-2,39)$ (30). Sin embargo, el efecto del CYP2C19 puede ser superado con dosis altas del IBP; por ejemplo, $40 \mathrm{mg}$ de omeprazol 2-3 veces al día o dosis equivalentes de otros IBP (21).

Vonoprazán es un nuevo IBP que inhibe la secreción de ácido bloqueando competitivamente la unión del potasio, en la adenosina-trifosfatasa (ATPasa) de la célula parietal (31). Supera algunos inconvenientes de los IBP convencionales tales como vida media corta, máxima inhibición de la secreción de ácido después de 4-5 dosis, necesidad de envoltura protectora del ácido y, adicionalmente, su actividad es independiente del polimorfismo de CYP2C19 $(32,33)$. En Japón está aprobado para el tratamiento de úlceras pépticas, enfermedad por reflujo gastroesofágico, profilaxis y tratamiento de lesiones gastroduodenales por antiinflamatorios no esteroideos (AINE), y para erradicar H. pylori $(34,35)$.

En un estudio japonés (35), la erradicación con un esquema que incluyó vonoprazán fue $92 \%$ frente a 75,9 \% en el grupo que recibió lansoprazol. Cuando se incluyó en terapias de segunda línea el éxito fue de 98,0 \% (35). Un metaanálisis reciente encontró que las terapias con vonoprazán son más eficaces que las que utilizan los IBP convencionales (36).

\section{PARTICULARIDADES DE LOS ANTIBIÓTICOS}

La variedad de antibióticos para erradicar $H$. pylori es pequeña, lo que varía son las combinaciones y sus dosis $(19,21-24,37)$. 


\section{Amoxicilina}

Es un derivado de la penicilina, inhibe la síntesis de la pared bacteriana y tiene vida media aproximada de una hora (21). Tiene efecto bactericida dependiente del tiempo y cuando se administra 3 o 4 veces al día logra una concentración mínima inhibitoria (MIC) adecuada y sostenida $(26,30)$. Estas características farmacocinéticas contrastan con la dosificación tradicional de 2 veces al día (26). La formulación adecuada debe ser 3 o 4 veces al día para mantener los niveles terapéuticos permanentemente $(21,26,30,38)$. La resistencia primaria de $H$. pylori a este antibiótico es muy rara a nivel mundial. En América Latina es $4 \%$ (39) y en Colombia $<2 \%(40)$. Por ese perfil, es un excelente medicamento que puede utilizarse incluso en terapias de segunda línea después de un esquema previo fallido en el cual se hubiera utilizado $(37,40)$.

\section{Claritromicina}

Es un macrólido que actúa uniéndose a la unidad 50S del ribosoma bacteriano (41). Desde hace varias décadas se utiliza a nivel mundial en la terapia triple estándar (TTE) (21-25). Su vida media es aproximadamente 5 horas y se puede administrar 2 veces al día (26). Por su amplia prescripción para múltiples enfermedades, $H$. pylori ha desarrollado una alarmante resistencia a nivel mundial (41). En Japón aumentó de 1,8 \% en 1996 a 27,1 \% en 2008 (42). En China la resistencia era de 14,8 \% en 2000 y en 2014 de 52,6 \% (41). En América Latina es en promedio $12 \%$ hasta 2011 (32) y en Colombia $25 \%$ (43). A comienzos del 2017, la OMS incluyó a H. pylori resistente a claritromicina en la lista de los 16 microorganismos que amenazan a la humanidad (44) y para los cuales se necesitan estrategias terapéuticas urgentes con el fin de erradicarlas.

\section{Metronidazol}

Es una prodroga que produce rupturas en la doble cadena de ácido desoxirribonucleico (ADN) bacteriano (9). Su vida media plasmática es cercana a 8 horas, por lo cual podría administrarse 2 o 3 veces al día (26). La resistencia primaria a este es muy alta en muchos países: Turquía, Irán, China y América Latina. (21). En dicho continente la resistencia promedio es de $53 \%$, siendo Colombia el país con la resistencia más alta reportada (83\%) (39). Es el único medicamento cuya resistencia in vitro puede vencerse aumentando la dosis y la duración del tratamiento (45). En terapia cuádruple, $500 \mathrm{mg} 4$ veces al día o $400 \mathrm{mg} 4$ veces al día durante 14 días logra un éxito de $92 \%$ aun con cepas resistentes (46), que es similar a la encontrada cuando no hay resistencia al mismo (47). Con esa posología, en regiones con alta resistencia al metronidazol las terapias cuádru- ples con bismuto tienen eficacia superior a $85 \%$ con una duración de 10-14 días (48-52).

\section{Quinolonas}

Estos medicamentos actúan en la $\mathrm{ADN}$ girasa causando rupturas en el ADN bacteriano (26). Su efecto bactericida depende más de la $\mathrm{C}_{\text {max }}$ que del tiempo por encima de la MIC, por lo cual la dosis puede ser una vez al día (26). Su principal representante es la levofloxacina, que se ha utilizado en esquemas de segunda línea o en primera línea cuando las tasas de resistencia a claritromicina son superiores a $15 \%$ o hay alergia a la penicilina $(40,46)$. Similar a la claritromicina, por su amplio uso en otras enfermedades especialmente otorrinolaringológicas, $H$. pylori ha desarrollado gran resistencia a nivel mundial (20). En América Latina la resistencia promedio es del $15 \%$ (39) y en Bogotá (Colombia) del $27 \%(48,49)$.

\section{Tetraciclina}

Sus representantes más importantes son la tetraciclina y doxiciclina. Actúan uniéndose a la subunidad $30 \mathrm{~S}$ del ribosoma bacteriano bloqueando la síntesis proteica (26). Su vida media aproximada es 6 horas, pudiéndose administrar 2 o 3 veces al día, aunque lo más utilizado es 3 a 4 veces al día (26). La resistencia primaria es muy infrecuente y no representa un inconveniente para su utilización (46). En América Latina la resistencia promedio es del $6 \%$ (39).

\section{Bismuto}

Las sales de este medicamento se utilizan en gastroenterología desde el siglo XIX (47), particularmente como tratamiento primario o adyuvante de la dispepsia y las úlceras pépticas, hasta su reemplazo posterior y paulatino por antiácidos (47). Las sales más populares son subcitrato, subnitrato y subsalicilato (47). Estas se hidrolizan en el estómago, formando polímeros insolubles con efecto bactericida, cuyo mecanismo de acción más importante para $H$. pylori es impedir el ingreso de hidrogeniones al citoplasma y, de esa manera, impedir su replicación $(50,51)$. Hasta el momento no se ha informado resistencia de $H$. pylori a este medicamento y su seguridad y tolerancia han sido demostradas $(38,50)$.

La terapia exitosa con este medicamento para erradicar H. pylori se desarrolló en Australia por Borody en 1989 (46). Ese esquema incluía 3 medicamentos: subcitrato de bismuto, metronidazol y tetraciclina (46). Posteriormente, se adicionó IBP y se extendió a 14 días, para recuperar la eficacia disminuida por la resistencia a metronidazol (47). La posología de este esquema es compleja por su duración y el 
gran número de tabletas al día: 2 tabletas de bismuto 4 veces al día, tetraciclina $500 \mathrm{mg} 4$ veces al día, metronidazol 500 mg 3 veces al día y el IBP 2 veces al día, lo cual incide notablemente en la adherencia al mismo. Adicionalmente, también produce efectos adversos, por lo cual se han implementado diferentes estrategias como la educación del paciente y disminución de la duración a 7-10 días, y también disminuyendo la dosis del metronidazol cuando la resistencia al mismo es baja y, finalmente, el desarrollo de una cápsula (PYLERA) que contiene los 3 medicamentos originales $(46,47)$. La eficacia esperada de la terapia cuádruple con bismuto por 14 días es superior a $95 \%$, aunque exista alta resistencia a metronidazol (52). Esta terapia cuádruple se recomienda como esquema de segunda línea o de primera línea cuando hay alergia a la penicilina, la resistencia a claritromicina es superior a $15 \%$ o cuando exista resistencia dual a claritromicina y metronidazol (46). También se recomienda como terapia de segunda de segunda línea cuando una terapia de primera línea ha fallado previamente (46).

Un uso reciente del bismuto es adicionarlo a las distintas terapias triples para contrarrestar las resistencias a claritromicina y a levofloxacina $(53,54)$. En la guía colombiana se recomienda esta estrategia (55). Los estudios iniciales con ese concepto se hicieron en Italia y posteriormente en España (56). En China (57) y en Turquía (58) no se ha demostrado la ventaja de adicionar bismuto a las terapias triples de primera línea cuando hay resistencia a claritromicina o a levofloxacina.

\section{Rifabutina}

Es un metabolito de la rifampicina, cercano a la rifamicina (59). Fue descubierto hace más de 20 años y usado en el tratamiento de erradicación de $H$. pylori en esquemas de cuarta línea o de salvamento (40, 59-61). Tiene baja biodisponibilidad y gran volumen de distribución (59). Se caracteriza por tener mayor capacidad de penetración intracelular y distribución tisular que la rifampicina, probablemente por ser más lipofílico (59). Actúa en la subunidad $\beta$ de la ARN-polimerasa dependiente del ADN bacteriano, inhibiendo la síntesis y transcripción de proteínas $(59,61,62)$. La resistencia de $H$. pylori a este medicamento es del $1 \%$ (50). Tiene como inconvenientes el alto costo y que no está fácilmente disponible en todas partes. Produce efectos adversos en más del $20 \%$ de los pacientes, siendo el más temido la mielotoxicidad, que se presenta con dosis de 600 mg al día o más y que desaparece al suspenderlo (63).

\section{Furazolidona}

Es un antimicrobiano de amplio espectro, perteneciente a la familia de los nitrofuranos (64). Inhibe la monoami- nooxidasa y es muy eficaz contra diversos microrganismos y muy económico (64-66). Su desventaja es su falta de disponibilidad (64-66). En gastroenterología se utilizó inicialmente para tratar enfermedades diarreicas (67). Tiene eficacia demostrada contra $H$. pylori, especialmente asociado con bismuto (67), aunque frecuentemente produce efectos adversos al interactuar con derivados de la soya y los quesos maduros; puede producir desde síntomas leves como náuseas, emesis y hasta crisis hipertensivas y convulsiones $(64,67)$. Este medicamento ha sido ampliamente usado en esquemas de tratamiento en China, Irán (64-68) y también en Colombia. La resistencia reportada en China es de $0 \%$ a $1 \%$ y en América Latina, particularmente en Brasil, de $3 \%$ en promedio $(39,67)$.

\section{DURACIÓN DE LOS ESQUEMAS DE ERRADICACIÓN}

La duración inicial de la TTE era 14 días, pero por la presión de la industria farmacéutica, se acortó a 7 días porque le traía ventajas comerciales. Sin embargo, se asoció con menor eficacia. Las revisiones sobre las terapias de 7 días indujeron a aumentar la duración a 10 días, aunque su éxito disminuyó progresivamente conforme aumentó la resistencia a claritromicina, metronidazol y últimamente a levofloxacina (46). En la última década su eficacia ha sido inaceptable $(<80 \%)$ (45). Desde el IV consenso de Maastricht se recomendó extender la duración a 14 días para aumentar la eficacia $5 \%$ en promedio (53). La mayoría de los estudios eran consistentes en la ventaja de una mayor duración (24). Un metaanálisis de Cochrane en 2013 (69) demostró que la TTE de 14 días era superior a las de 7-10 días con una diferencia del $10 \%$ (69). Conforme se extendió a más días, también aumentaron los eventos adversos de $15,5 \%$ a $19,4 \%$, aunque no se tradujo en abandonos del tratamiento (69). En otros tipos de esquemas, también se ha demostrado mayor éxito con la duración de 14 días frente a terapias más cortas, excepto probablemente para la terapia cuádruple con bismuto, cuya duración de 10 días que sigue siendo eficaz (70). La recomendación actual en los consensos internacionales más importantes sobre erradicación de $H$. pylori $(52,54,71)$ y de la guía colombiana (55) es que la duración para todas las terapias sea de 14 días, excepto los que contienen rifabutina, que pueden ser de 10 días $(38,53,54,70,71)$.

\section{TERAPIAS DE ERRADICACIÓN: ¿CUÁL PRIMERO?}

Idealmente, el tratamiento de $H$. pylori debería ser similar a cualquier enfermedad infecciosa, basada en pruebas de susceptibilidad (16). Sin embargo, los cultivos de biopsias gástricas o métodos moleculares (reacción en cadena de la polimerasa $[\mathrm{PCR}]$, hibridación in situ) para investigar la 
susceptibilidad $(55,72,73)$ no están disponibles en todos los sitios y esto obliga a iniciar el tratamiento empíricamente $(46,47)$. Ante esta realidad, la recomendación es que la elección de las terapias debe tener en cuenta el patrón de resistencia local a los antibióticos e idealmente que la eficacia de los esquemas utilizados haya sido demostrada independientemente de consensos o guías internacionales. Los tratamientos elegidos deben tener eficacia mínima del $90 \%$ (74). Hasta el momento, ningún esquema es $100 \%$ eficaz, por lo cual se necesitan tratamientos de segunda, tercera línea y de rescate o salvamento (40). Los diferentes tipos de esquemas se describen a continuación.

\section{Terapia cuádruple clásica}

Su duración es de 14 días. Consta de IBP 2 veces al día + bismuto subsalicilato ( $550 \mathrm{mg} 4$ veces/día o 2 veces al día) + metronidazol (500 mg 3 veces/día) + tetraciclina $\mathrm{HCl}$ (500 mg 4 veces/día) $(11,46,53,54,56,70,71)$. El bismuto se puede dar 2 veces al día.

\section{Terapia híbrida}

Se compone de 2 fases de 7 días de duración cada una (11, $66,74)$. En los primeros 7 días: amoxicilina en dosis estándar u optimizadas ( 3 o 4 veces al día) más IBP 2 veces al día y en la última semana se adicionan 2 antibióticos diferentes, usualmente claritromicina $(500 \mathrm{mg})+$ metronidazol/ tinidazol $(500 \mathrm{mg})$, cada uno 2 veces al día. Su eficacia es del $97 \%$ (75). Cuando hay resistencia combinada a claritromicina y a metronidazol superior al $9 \%$ (resistencia dual), la eficacia es menor al $90 \%$ (66). Recientemente, en Colombia se investigó una terapia híbrida de 15 días que logró una eficacia de $94 \%$ (76). La posología de esta terapia es la siguiente: amoxicilina $500 \mathrm{mg} 4$ veces/día + esomeprazol $40 \mathrm{mg} 2$ veces/día 15 días. En los últimos diez días se administra subsalicilato de bismuto 2 tabletas 2 veces/día + doxiciclina $\left(\right.$ Vibramicina $\left.^{\oplus}\right) 100 \mathrm{mg} 2$ veces/día.

\section{Terapia concomitante}

Este es el esquema más utilizado, combina los IBP con amoxicilina, claritromicina y metronidazol $(46,53,54$, $70,71)$. Es recomendada porque la resistencia dual a claritromicina y metronidazol es infrecuente $(26,46,53,54$, $70,71)$. Cuando hay resistencia dual, la eficacia disminuye notablemente $(66,74,77)$. La eficacia global es $88 \%-90 \%$ $(38,46)$. Como se ha comentado, la adición de bismuto a las distintas terapias triples para contrarrestar las resistencias a claritromicina y a levofloxacina $(53,54)$ ha sido una nueva estrategia que inició en Europa (56) y actualmente es válida y recomendada en la guía colombiana (55).

\section{Terapia triple estándar}

Este esquema fue muy popular en las últimas décadas; incluye IBP, amoxicilina y claritromicina (53-55, 70, 71). Sin embargo, en la actualidad para el uso de dicho régimen terapéutico, este debe administrarse durante 14 días; lo anterior es válido cuando el nivel de resistencia de claritromicina local es $<15 \%$. El esquema sería: amoxicilina 3-4 veces/día + IBP 2 veces/día + claritromicina 500 mg 2 veces/día (53-55). Si la resistencia es $>15 \%$, se debe adicionar bismuto subsalicilato (Bisbacter $\left.{ }^{\circledast}\right) 2$ tabletas masticadas antes del desayuno y de la cena. O cambiar la claritromicina por levofloxacina $500 \mathrm{mg}$ al día. Si la resistencia a levofloxacina es $>20 \%$, se podría mantener, pero adicionando bismuto en la misma posología descrita anteriormente.

\section{ELECCIÓN PARA PRIMERA, SEGUNDA Y TERCERA LÍNEA}

\section{Tratamientos de primera línea}

Es el esquema terapéutico que se da inicialmente. Como se mencionó, debería basarse en pruebas de susceptibilidad $(10,46)$. Cuando se hace de esa manera, la eficacia por intención de tratar (ITT) es de 94,7 \% (IC $95 \%: 88,8$ $\%-100 \%$ ) y por protocolo (PP) es de 96,4 \% (IC $95 \%$ : 91,5\%-100 \%) frente a 71,9\% (IC $95 \%: 60,2 \%-83,5 \%$ ) y 73,2 \% (IC $95 \%: 61,5 \%-84,8 \%$ ) con las terapias empíricas (78). Cuando la resistencia a claritromicina es $<15$ $\%$ se puede utilizar la terapia triple con claritromicina más amoxicilina e IBP por 14 días (53). Si es $>15 \%$ se recomienda remplazar este medicamento por levofloxacina o como alternativa adicionar bismuto $(53,54,70,71), 2$ tabletas de subsalicilato (Bisbacter ${ }^{\circledR}$ ) masticadas antes del desayuno y de la cena.

En Colombia, la tasa de resistencia a claritromicina es de $20,5 \%$ (79), a levofloxacina $27,3 \%(80)$ y a metronidazol $>80 \%$ (43). Con este perfil de resistencia en particular, la recomendación es adicionar a las terapias triples de 14 días subsalicilato de bismuto, 2 tabletas 2 veces al día. Las terapias cuádruples con bismuto no necesitan pruebas de susceptibilidad (52).

\section{Tratamientos de segunda y de tercera línea}

Cuando fracasa el tratamiento de primera línea, el de segunda línea se elige entre cualquiera de los esquemas que no tengan los antibióticos utilizados en la de primera línea. De la misma forma, también se eligen los tratamientos de tercera línea. Otras terapias elegibles pueden ser la cuádruple clásica, concomitantes o híbridas (40). 


\section{Tratamientos de rescate (salvamento)}

Estos tratamientos son de cuarta línea, cuando 3 tratamientos previos han fallado (40). Los antibióticos para estos esquemas son furazolidona o rifabutina $(53,54,70,71,81)$.

Con furazolidona, la dosis recomendada es $100 \mathrm{mg} 3$ veces al día por 14 días $(43,64-66)$. Se utiliza en terapias cuádruples con bismuto ( 2 a 4 veces al día), con amoxicilina o tetraciclina más IBP $(43,64-66)$. Este medicamento ya se utilizó en Colombia en terapia cuádruple por 14 días y la tasa de erradicación fue de $86 \%$ (IC $95 \%$ : $65 \%$-94 $\%)$ con amoxicilina (82) y $91 \%$ con tetraciclina (83). El esquema sería de la siguiente manera: Amoxicilina $850 \mathrm{mg}$ 3 veces al día (o $500 \mathrm{mg}$ cada 6 horas) más furazolidona 100 mg 3 veces al día más subsalicilato de bismuto 2 tabletas con el desayuno y con la cena, más IBP 2 veces al día. En los alérgicos a la penicilina, se cambiaría la amoxicilina por tetraciclina $500 \mathrm{mg}$ cada 6 horas.

No obstante su eficacia, la furazolidona es subutilizada por el temor a sus efectos adversos. Además, se ha especulado que podría tener efectos oncogénicos $(63,65)$; sin embargo, no hay evidencias de esa posibilidad $(66,84,85)$. Al respecto, la Agencia Internacional de Investigación en Cáncer de la OMS (IARC) la tiene incluida en la categoría 3, es decir, "No clasificable como carcinógeno para los humanos" (84). Estudios más recientes han ratificado su seguridad $(46,64,84)$.

\section{Rifabutina}

El esquema con este medicamento es el siguiente: rifabutina $150 \mathrm{mg} 2$ veces/día, más amoxicilina más IBP por 10 días (86). Esta triple terapia tiene eficacia del 79\% (55), cuando se utiliza como recate después de 3 o 4 terapias previas fallidas. La adición de bismuto aumenta la eficacia a 96,6 $\%$ (87). La rifabutina se decide en pacientes con patologías importantes o serias en quienes las terapias previas hallan fallado, tales como úlceras pépticas complicadas, linfoma MALT gástrico o antecedente de CG (55). Un flujograma sugerido para tratar $H$. pylori se muestra en la Figura 1 y los respectivos esquemas mencionados en el mismo son los descritos previamente.

\section{Probióticos}

Los probióticos son microorganismos vivos o sustancias producidas por estos que tienen efectos benéficos (21, $88)$. Se han utilizado como adyuvantes en la terapia antibiótica de erradicación de $H$. pylori. Se les atribuyen beneficios tales como la estimulación de la respuesta inmune, modulación de la microbiota y disminución de efectos adversos de los antibióticos (21). La especie más estudiada es Lactobacillus spp., que en algunos estudios ha mejorado la tasa de eficacia (88). Otros estudios y metaanálisis han confirmado el beneficio de los probióticos incluyendo también Biffidobacterium spp. y Saccharomyces boulardii (88, 89). Este último tipo de probióticos ha llamado la atención recientemente, se ha evidenciado en un metaanálisis realizado en 2015 con más de 2200 participantes, en el grupo de suplementación con estos microorganismos hubo un riesgo relativo (RR) de éxito en la erradicación de $H$. pylori de 1,11 (IC $95 \%$ : 1,06-1,17); y RR de efectos adversos relacionados con la terapia de 0,44 (IC $95 \%$ : 0,31-0,64), específicamente diarrea: 0,51 (IC $95 \%$ : 0,42-0,62). (89) El mecanismo de acción de estos no es del todo claro y hasta ahora se han estudiado múltiples cepas, dosis, tiempos de duración de la terapia probiótica como coadyuvante (90).

Actualmente se tiene un escenario poco claro en relación con una recomendación uniforme de estos productos. En el consenso de Maastricht se consideró que solo algunas cepas específicas de los probióticos antes nombrados son útiles para incrementar la eficacia y reducir la ocurrencia de efectos adversos relacionados con los antibióticos (52). Sin embargo, ni en el consenso de Toronto ni en la guía española se recomiendan los probióticos $(54,71)$.

\section{Importancia del biofilm}

Similar a otros microorganismos como Pseudomonas aeruginosa, Staphylococcus aureus, entre otras, H. pylori produce biofilm, que consiste en agregados de organismos que crecen unidos entre sí, aprovechando las superficies o interfaces (91). El biofilm es un componente antiguo e integral en el ciclo de vida procariota y en la actualidad es visto como un factor de virulencia independiente, siendo reconocido como causa de exacerbación de infecciones respiratorias crónicas (4). El biofilm favorece notablemente a $H$. pylori: dificulta la llegada de los antibióticos a la bacteria, disminuye su replicación por la limitación de nutrientes y lo protege contra la respuesta inmune celular y humoral (91).

Para debilitar la protección del biofilm de H. pylori, se ha utilizado el mucolítico $\mathrm{N}$-acetilcisteína (NAC) antes de iniciar los antibióticos (92) En un ensayo clínico sin cegamiento publicado en 2010 se usó esta estrategia cuando un tratamiento previo había fallado y logró una eficacia del $65 \%$ en el grupo de NAC frente al $20 \%$ en el grupo control (93). Otras estrategias han sido utilizar otras sustancias y enzimas degradantes del biofilm en estudios in vitro, con resultados aún pendientes por validar en ensayos clínicos (4).

\section{Vacunas}

La vacunación ha surgido como una nueva alternativa en la lucha contra la resistencia bacteriana, pero enfocada en la prevención (94). Contra H. pylori se han probado vacunas en modelos animales logrando una protección aceptable (23). En humanos, los resultados son variables, 


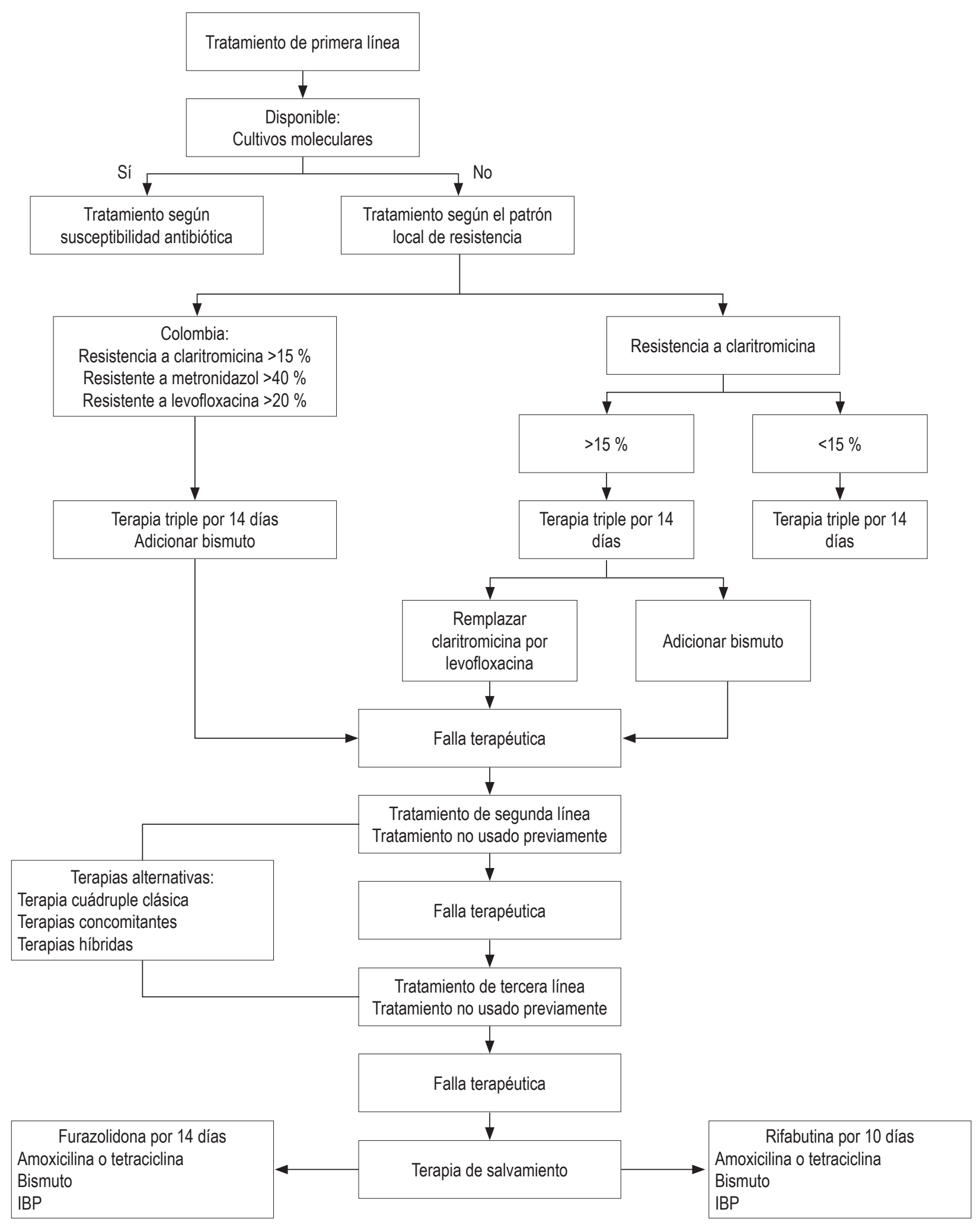

Figura 1. Flujograma sugerido para tratar H. pylori. 
dependiendo de la ruta de la inmunización y los antígenos utilizados (95). Recientemente, en el primer ensayo clínico aleatorizado doble ciego se obtuvo eficacia en la inmunogenicidad en el $71 \%$ a 1 año y $55 \%$ a 3 años (96). Hasta el momento no se dispone de una vacuna definitiva (97).

\section{Conflicto de intereses}

Ninguno.

\section{REFERENCIAS}

1. Arévalo A, Trespalacios AA, Otero W. Importancia de la proteína CagA en la infección por Helicobacter pylori. Rev. Col Gastroenterol 2009; 24 (4): 388-95.

2. Trespalacios AA, Otero W, Caminos J, et al. Phenotypic and Genotypic Analysis of Clarthromycin-Resistant Helicobacter pylori from Bogotá D.C., Colombia. J Microbiol. 2013; 51 (4): 448-452. doi: 10.1007/s12275-013-2465-6.

3. Otero W, Trespalacios AA, Otero E. Helicobacter pylori: Tratamiento actual. Un importante reto en gastroenterología. Rev Colomb Gastroenterol. 2009; 24(3):279-92.

4. Cammarota G, Sanguinetti M, Gallo A, Posteraro B. Review article: biofilm formation by Helicobacter pylori as a target for eradication of resistant infection. Aliment Pharmacol Ther 2012; 36 (3): 222-230. doi: 10.1111/j.1365-2036.2012.05165.x.

5. Hooi JKY, Lai WY, Ng WK, Suen MMY, Underwood FE, Tanyingoh D, et al. Global Prevalence of Helicobacter pylori Infection: Systematic Review and Meta-Analysis. Gastroenterology. 2017;153(2):420-429. doi: 10.1053/j. gastro.2017.04.022.

6. Cosme A, Montes M, Martos M, Gil I, Mendarte U, Salicio Y, et al. Usefulness of antimicrobial susceptibility in the eradication of Helicobacter pylori. Clinical Microbiology and Infection 2013;19(4):379-83. doi: 10.1111/j.1469-0691.2012.03844.x.

7. Peleteiro B, Bastos A, Ferro A, Nuno L. Prevalence of Helicobacter pylori Infection Worldwide: A Systematic Review of Studies with National Coverage. Dig Dis Sci 2014; 59 (8): 1698-1709. doi: 10.1007/s10620-014-3063-0.

8. Axon A. Helicobacter pylori and Public Health. Helicobacter 2014;19(Suppl 1): 68-73. doi: 10.1111/hel.12155.

9. Gisbert JP. Enfermedades relacionadas con la infección por Helicobacter pylori. Gastroenterol Hepatol. 2015;38(Supl 1):39-48. doi: 10.1016/S0210-5705(15)30018-2.

10. Gisbert JP. Enfermedades relacionadas con la infección por Helicobacter pylori. Gastroenterol Hepatol. 2014;37(Supl 3):40-52. doi: 10.1016/S0210-5705(14)70082-2.

11. Marshall BJ, Warren JR. Unidentified curved bacilli in the stomach of patients with gastritis and peptic ulceration. Lancet. 1984;1:1311-5. doi: 10.1016/S0140-6736(84)91816-6.

12. Kyle R, Steensma D, Shampo M. Barry James MarshallDiscovery of Helicobacter pylori as a Cause of Peptic Ulcer. Mayo Clin Proc. 2016;91(5):e67-e68. doi: 10.1016/j. mayocp.2016.01.025.
13. Otero W, Gómez M, Trespalacios AA. Helicobacter pylori: después de todo. Temas escogidos de gastroenterología. Asociación Colombiana de Gastroenterología. 2007;43-56.

14. Urrego J, Otero W, Gomez M. Helicobacter prlori y enfermedades hematológicas. Rev Col Gastroenterol. 2013;28(4):329-37.

15. Mégraud F, Bessède E, Varon C, Helicobacter pylori infection and gastric carcinoma. Clin Microbiol Infect 2015;21(11):984-90. doi: 10.1016/j.cmi.2015.06.004.

16. Graham DY. Helicobacter pylori Update: Gastric Cancer, Reliable Therapy, and Possible Benefits. Gastroenterol 2015;148(4):719-31. doi: 10.1053/j.gastro.2015.01.040.

17. IARC Working Group on the Evaluation of Carcinogenic Risks to Humans. Schistosomes, liver flukes and Helicobacter pylori. Lyon, 7-14 June 1994. IARC Monogr Eval Carcinog Risks Hum. 1994;61:1-241.

18. Gomez M, Ruiz O, Hernandez D, Albis R, Otero W, Sabbagh L. Erradicación del Helicobacter pylori: encuesta realizada por la Asociación Colombiana de Gastroenterología. Rev. Col Gastroenterol. 2015;30(1):25-31. doi: 10.22516/25007440.19.

19. Malfertheiner P, Venerito M, Selgrad M. Helicobacter pylori infection: selected aspects in clinical management. Curr Opin Gastroenterol. 2013;29(6):669-75. doi: 10.1097/ MOG.0b013e328365d443.

20. Sugano K, Tack J, Kuipers EJ, Graham DY, El-Omar EM, Miura S, et al. Kyoto global consensus report on Helicobacter pylori gastritis. Gut. 2015;64(9):1353-67. doi: 10.1136/ gutjnl-2015-309252.

21. Molina J, Shiotani A. Practical Aspects in Choosing a Helicobacter pylori Therapy. Gastroenterol Clin N Am 2015; 44:519-535. doi: 10.1016/j.gtc.2015.05.004.

22. Graham DY, Fischbach L. Helicobacter pylori treatment in the era of increasing antibiotic resistance. Gut 2010; 59: 1143-1153. doi: 10.1136/gut.2009.192757.

23. Malfertheiner P, Link A, Selgrad M. Helicobacter pylori: perspectives and time trends. Nat Rev Gastroenterol Hepatol. 2014;11(10):628-38. doi: 10.1038/nrgastro.2014.99.

24. Molina J, Gisbert JP. Optimizing clarithromycin-containing therapy for Helicobacter pylori in the era of antibiotic resistance. World J Gastroenterol. 2014;20(30):10338-47. doi: 10.3748/wjg.v20.i30.10338.

25. Sugimoto M, Furuta T. Efficacy of tailored Helicobacter pylori eradication therapy based on antibiotic susceptibility and CYP2C19 genotype. World J Gastroenterol. 2014;20(21):6400-11. doi: 10.3748/wjg.v20.i21.6400.

26. Furuta T, Graham DY. Pharmacologic Aspects of Eradication Therapy for Helicobacter pylori Infection. Gastroenterol Clin N Am. 2010;39(3):465-80. doi: 10.1016/j.gtc.2010.08.007.

27. Kim SY, Choi DJ, Chung JW. Antibiotic treatment for Helicobacter pylori: Is the end coming? World J Gastrointest Pharmacol Ther. 2015;6(4):183-98. doi: 10.4292/wjgpt. v6.i4.183.

28. Furuta T, Ohashi K, Kamata T, Takashima M, Kosuge K, Kawasaki T, et al. Effect of Genetic Differences in Omeprazole Metabolism on Cure Rates for Helicobacter pylori Infection 
and Peptic Ulcer. Ann Intern Med. 1998;129(12):1027-30. doi: 10.7326/0003-4819-129-12-199812150-00006.

29. Tang HL, Li Y, Hu YF, Xie HG, Zhai SD. Effects of CYP2C19 loss-of-function variants on the eradication of H. pylori infection in patients treated with proton pump inhibitor-based triple therapy regimens: a meta-analysis of randomized clinical trials. PLOS ONE. 2013;8(4):e62162. doi: 10.1371/journal.pone.0062162.

30. Lee JY, Kim N, Kim MS, Choi YJ, Lee JW, Yoon H, et al. Factors affecting first-line triple therapy of Helicobacter pylori including CYP2C19 genotype and antibiotic resistance. Dig Dis Sci. 2014;59:1235-43. doi: 10.1007/s10620-014-3093-7.

31. Echizen $H$. The first-in-class potassium-competitive acid blocker, vonoprazan fumarate: Pharmacokinetic and pharmacodynamic considerations. Clin Pharmacokinet. 2016;55:409-18. doi: 10.1007/s40262-015-0326-7.

32. Hori $Y$, Imanishi A, Matsukawa J, Tsukimi $Y$, Nishida $H$, Arikawa $\mathrm{Y}$, et al. 1-[5-(2-Fluorophenyl)-1-(pyridin-3-ylsulfonyl)-1Hpyrrol-3-yl]-N-methylmethanamin e monofumarate (TAK438), a novel and potent potassium-competitive acid blocker for the treatment of acid-related diseases. J Pharmacol Exp Ther. 2010;335(1):231-8. doi: 10.1124/jpet.110.170274.

33. Sakurai Y, Nishimura A, Kennedy G, Hibberd M, Jenkins R, Okamoto H, et al. Safety, tolerability, pharmacokinetics, and pharmacodynamics of single rising TAK-438 (Vonoprazan) doses in healthy male Japanese/ non-Japanese subjects. Clin Transl Gastroenterol 2015; 6:e94. doi: 10.1038/ctg.2015.18

34. Garnock-Jones KP. Vonoprazan: first global approval. Drugs. 2015;75(4):439-43. doi: 10.1007/s40265-015-0368-z.

35. Murakami K, Sakurai Y, Shiino M, Funao N, Nishimura A, Asaka M. Vonoprazan, a novel potassium-competitive acid blocker, as a component of first-line and second-line triple therapy for Helicobacter pylori eradication: a phase III, randomised, double-blind study. Gut. 2016;65(9):1439-46. doi: 10.1136/gutjnl-2015-311304.

36. Jung YS, Kim EH, Park CH. Systematic review with metaanalysis: the efficacy of vonoprazan-based triple therapy on Helicobacter pylori eradication. Aliment Pharmacol Ther.. 2017;46(2):106-14. doi: 10.1111/apt.14130.

37. Malfertheiner P, Selgrad M. Helicobacter pylori. Curr Opin Gastroenterol. 2014;30(6):589-95. doi: 10.1097/ MOG.0000000000000128.

38. Gisbert JP, McNicholl AG. Optimization strategies aimed to increase the efficacy of $\mathrm{H}$. pylori eradication therapies. Helicobacter.. 2017; 22(4). doi: 10.1111/hel.12392.

39. Camargo MC, García A, Riquelme A, et al. The Problem of Helicobacter pylori Resistance to Antibiotics: A Systematic Review in Latin America. Am J Gastroenterol. 2014;109(4):485-95. doi: 10.1038/ajg.2014.24.

40. Otero W, Otero L, Gómez M. Helicobacter pylori: tratamiento en 2018. En: Gómez M (editor). Temas Escogidos de Gastroenterología. 2018. p. 63-78.

41. Thung I, Aramin H, Vavinskaya V, Gupta S, Park JY, Crowe SE, et al. Review article: the global emergence of Helicobacter pylori antibiotic resistance. Aliment Pharmacol Ther. 2016;43(4):514-33. doi: 10.1111/apt.13497.
42. Horiki N, Omata F, Uemura M, Suzuki S, Ishii N, Iizuka Y, et al. Annual change of primary resistance to clarithromycin among Helicobacter pylori isolates from 1996 through 2008 in Japan. Helicobacter. 2009;14(5):86-90. doi: 10.1111/j.1523-5378.2009.00714.x.

43. Trespalacios AA, Otero W, Mercado M, et al. Impacto de la resistencia de Helicobacter pylori a los antimicrobianos en la eficacia de la terapia triple estándar y en dos triples terapias con levofloxacina en pacientes colombianos. Gastroenterol Latinoam. 2012;23:S35.

44. World Health Organization. WHO publishes list of bacteria for which new antibiotics are urgently needed. WHO [internet] 2017 [acceso el 20 de diciembre de 2017]. Disponible en: www.who.int/entity/mediacentre/news/ releases $/ 2017 /$ bacteria-antibiotics-needed/en/.

45. Wu JY, Liou JM, Graham DY. Evidence-based recommendations for successful Helicobacter pylori treatment. Expert Rev Gastroenterol Hepatol. 2014;8(1):21-8. doi: 10.1586/17474124.2014.859522.

46. Graham DY, Dore MP. Helicobacter pylori therapy: a paradigm shift, Expert Review of Anti-infective Therapy 2016, 14(6):577-85. doi: 10.1080/14787210.2016.1178065.

47. Graham DY, Lee SY. How to Effectively Use Bismuth Quadruple Therapy: The Good, the Bad, and the Ugly. Gastroenterol Clin North Am. 2015; 44: 537-563. doi: 10.1016/j.gtc.2015.05.003.

48. Trespalacios-Rangél AA, Otero W, Arévalo-Galvis A, Poutou-Piñales RA, Rimbara E, Graham DY. Surveillance of Levofloxacin Resistance in Helicobacter pylori Isolates in Bogotá-Colombia (2009-2014). PLoS One. 2016;11(7):e0160007. doi: 10.1371/journal.pone.0160007.

49. Sugimoto M, Sahara S, Ichikawa H, Kagami T, Uotani T, Furuta T. High Helicobacter pylori cure rate with sitafloxacin-based triple therapy. Aliment Pharmacol Ther. 2015;42(4):477-83. doi: 10.1111/apt.13280.

50. Marcus EA, Sachs G, Scott DR. Eradication of Helicobacter pylori infection. Current gastroenterology reports. 2016;18(7):33. doi: 10.1007/s11894-016-0509-x.

51. Keogan DM, Griffith DM. Current and Potential Applications of Bismuth-Based Drugs. Molecules 2014; 19: 15258-97. doi: 10.3390/molecules 190915258.

52. Malfertheiner P, Megraud F, O’Morain CA, Gisbert JP, Kuipers EJ, Axon AT, et al. Management of Helicobacter pylori infection-the Maastricht V/Florence Consensus Report. Gut. 2017;66(1):6-30. doi: 10.1136/gutjnl-2016-312288.

53. Malfertheiner P, Megraud F, O'Morain CA, Atherton J, Axon AT, Bazzoli F, et al. Management of Helicobacter pylori infection-the Maastricht IV/ Florence Consensus Report. Gut. 2012;61(5):646-64. doi: 10.1136/gutjnl-2012-302084.

54. Gisbert JP, Molina-Infante J, Amador J, Bermejo F, Bujanda L, Calvet X, et al. IV Conferencia Española de Consenso sobre el tratamiento de la infección por Helicobacter pylori. Gastroenterol Hepatol. 2016;39(10):697-721. doi: 10.1016/j.gastrohep.2016.05.003.

55. Otero W, Trespalacios AA, Otero L, Vallejo M, Torres M, Pardo R, et al. Guía de práctica clínica para el diagnóstico y 
tratamiento de la infección por Helicobacter pylori en adultos. Rev Col Gastroenterol. 2015;30(Suppl 1):17-33.

56. Dore MP, Lu H, Graham DY. Role of bismuth in improving Helicobacter pylori eradication with triple therapy. Gut. 2016;65(5):870-8. doi: 10.1136/gutjnl-2015-311019.

57. Zhang W, Chen Q, Lian X, et al. Bismuth, lansoprazole, amoxicillin and metronidazole or clarithromycin as first-line Helicobacter pylori therapy. Gut. 2015;64:1715-20. doi: 10.1136/gutjnl-2015-309900.

58. Gokcan H, Oztas E, Onal IK. Different bismuth-based therapies for eradicating Helicobacter pylori: Randomized clinical trial of efficacy and safety. Clin Res Hepatol Gastroenterol. 2016;40:124-31. doi: 10.1016/j.clinre.2015.06.014.

59. O'Morain, C. and Montague, S. Challenges to Therapy in the Future. Helicobacter. 2000;5:23-6. doi: 10.1046/j.15235378.2000.0050S1023.x.

60. Perri F, Festa V, Andriulli A. Treatment of antibiotic-resistant Helicobacter pylori. N Engl J Med 1998; 339:53. doi: 10.1056/NEJM199807023390116.

61. Crabol Y, Catherinot E, Veziris N, et al. Rifabutin: where do we stand in 2016? J Antimicrob Chemother. 2016;71(7):1759-71. doi: 10.1093/jac/dkw024.

62. Della Bruna C, Schioppacassi G, Ungheri D, et al. LM 427, a new spiropiperidylrifamycin: in vitro and in vivo studies. $\mathrm{J}$ Antibiot (Tokyo). 1983;36:1502-6. doi: 10.7164/antibiotics.36.1502.

63. Gisbert JP, Calvet X. Review article: rifabutin in the treatment of refractory Helicobacter pylori infection. Aliment Pharmacol Ther. 2012;35:209-21. doi: 10.1111/j.13652036.2011.04937.x.

64. Graham DY, Lu H. Furazolidone in Helicobacter pylori therapy: misunderstood and often unfairly maligned drug told in a story of French bread. Saudi J Gastroenterol. 2012; 18:1-2. doi: 10.4103/1319-3767.91724.

65. Cheng H, HU FL.Furazolidone, amoxicillin, bismuth and rabeprazole quadruple rescue therapy for the eradication of Helicobacter pylori. World J Gastroenterol. 2009;15:86064. doi: 10.3748 /wjg. 15.860 .

66. Lu H, Zhang W, Graham DY. Bismuth containing quadruple therapy for Helicobacter pylori: lessons from China. Eur J Gastroenterol Hepato 2013;25:1134-40. doi: 10.1097/ MEG.0b013e3283633b57.

67. MohammadiM,Attaran B, Malekzadeh R, etal.Furazolidone, an Underutilized Drug for H. pylori Eradication: Lessons from Iran. Dig Dis Sci. 2017;62(8):1890-6. doi: 10.1007/ s10620-017-4628-5.

68. Hu Y, Zhu Y, Lu N-H. Novel and Effective Therapeutic Regimens for Helicobacter pylori in an Era of Increasing Antibiotic Resistance. Front. Cell. Infect. Microbiol. 2017; 7:168. doi: 10.3389/fcimb.2017.00168.

69. Yuan Y, Ford AC, Khan KJ, et al. Optimum duration of regimens for Helicobacter pylori eradication. Cochrane Database Syst Rev. 2013;12:CD008337. doi: 10.1002/14651858. CD008337.pub2.
70. Chey WD, Leontiadis GI, Howden CW, et al. ACG clinical guideline: treatment of Helicobacter pylori infection. Am J Gastroenterol 2017;112:212-39. doi: 10.1038/ajg.2016.563.

71. Fallone C, Chiba N, van Zanten S, Fischbach L, Gisbert JP, Hunt $\mathrm{R}$, et al. The Toronto consensus for the treatment of Helicobacter pylori infection in adults, Gastroenterology 2016; 151, 51-69.e14. doi: 10.1053/j.gastro.2016.04.006.

72. Calvet X. Diagnosis of Helicobacter pylori Infection in the proton pump Inhibitor era. Gastroenterol Clin N Am. 2015;44(3):507-18. doi: 10.1016/j.gtc.2015.05.001.

73. Malfertheiner P, Mégraud F, O’Morain C, et al. Current European concepts in the management of Helicobacter pylori infection-the Maastricht Consensus Report. The European Helicobacter pylori Study Group (EHPSG). Eur J Gastroenterol Hepatol. 1997;9:1-2. doi: 10.1097/00042737-199701000-00002.

74. Graham DY, Lee YC, Wu MS. Rational Helicobacter pylori therapy: evidence-based medicine rather than medicinebased evidence. Clin Gastroenterol Hepatol. 2014;12:177186. doi: 10.1016/j.cgh.2013.05.028.

75. Hsu PI,Wu DC, WuJH, et al.Modified sequential Helicobacter pylori therapy: proton pump inhibitor and amoxicillin for 14 days with clarithromycin and metronidazole added as a quadruple (hybrid) therapy for the final 7 days. Helicobacter 2011;16:139-45. doi: 10.1111/j.1523-5378.2011.00828.x.

76. Otero W, Gutiérrez O, Saabagh LC: Efectiveness of a hibrid therapy in eradicating Helicobacter pylori in a Colombian population. Gastroenterology. 2016;150:S246. doi: 10.1016/S0016-5085(16)30889-7.

77. Ducoumau A, Bénéjat L, Sifré LE, Lehours P, Mégraud F. Helicobacter pylori resistance to antibiotics in 2014 in France detected by phenotypic and genotypic methods. Clin Microbiol Infect 2016;22:715-8. doi: 10.1016/j. cmi.2016.06.003.

78. Park CS, Lee SM, Park CH, et al. Pretreatment Antimicrobial Susceptibility-Guided Vs. Clarithromycin-Based Triple Therapy for Helicobacter pylori Eradication in a Region With High Rates of Multiple Drug Resistance. Am J Gastroenterol. 2014;109:1595-602. doi: 10.1038/ajg.2014.222.

79. Arévalo A, Trespalacios AA, Otero W. Personalized therapy for Helicobacter pylori: influence of cyp2c19 genotype in first triple therapy. Sometido y aceptado para publicación. Helicobacter. 2019.

80. Trespalacios-Rangél AA, Otero W, Arévalo-Galvis A, Poutou-Piñales RA, Rimbara E, Graham DY. Surveillance of Levofloxacin Resistance in Helicobacter pylori Isolates in Bogotá-Colombia (2009-2014). PLoS One. 2016;11(7):e0160007. doi: 10.1371/journal.pone.0160007.

81. Villoria A, García P, Calvet X, Gisbert J, Vergara M. Metaanalysis: high dose proton pump inhibtor vs standard dose in triple therapy for Helicobacter pylori eradication. Aliment Pharmacol Ther. 2008;28:868-77. doi: 10.1016/ S0016-5085(08)61572-3.

82. Segura AM, Gutiérrez O, Otero W, Angel LA, Genta R, Graham DY. Furazolidone, amoxycillin, bismuth triple therapy 
for Helicobacter pylori infection. Aliment Pharmacol Ther 1997;11:529-32. doi: 10.1046/j.1365-2036.1997.00172.x.

83. Otero W, Gutierrez O, Sierra F. Erradicación de H pylori con Terapia Triple: Bismuto, Furazolidona, Tetraciclina. Acta Med Col. 1996;21:218 (Res).

84. Some food additives, feed additives and naturally occurring substances. IARC Monogr Eval Carcinog Risk Chem Hum. 1983;31:1-291.

85. Auro A, Sumano H, Ocampo L, Barragán A. Evaluation the carcinogenic effects of furazolidone and its metabolites in two fish species. Pharmacogenomics J. 2004;4:24-8. doi: $10.1038 /$ sj.tpj.6500216.

86. Sun Q, Liang X, Zheng Q. High efficacy of 14 -day triple therapy-based, bismuth-containing quadruple therapy for initial Helicobacter pylori eradication. Helicobacter 2010;15:233-8. doi: 10.1111/j.1523-5378.2010.00758.x.

87. Ciccaglione AF, Tavani R, Grossi L, et al. Rifabutin containing triple therapy and rifabutin with bismuth Containing quadruple therapy for third-line treatment of Helicobacter pylori infection: Two pilot studies. Helicobacter. 2016;21:375-81. doi: 10.1111/hel.12296.

88. O’Connor A., Vaira D., Gisbert JP, O’Morain C. Treatment of Helicobacter pylori infection 2014. Helicobacter. 2014;19(Suppl 1):38-45. doi: 10.1111/hel.12163.

89. Szajewska H, Horvath A, Kołodziej M. Systematic review with meta-analysis: Saccharomyces boulardii supplementation and eradication of Helicobacter pylori infection. Aliment Pharmacol Ther 2015;41:1237-45. doi: 10.1111/apt.13214.

90. Luther J, Chey WD, Saad RJ. A clinician's guide to salvage therapy for persistent Helicobacter pylori infection.
Hosp Pract (1995). 2011;39:133-140. doi: 10.3810/ hp.2011.02.383.

91. Yonezawa H, Osaki T, Kamiya S. Biofilm Formation by Helicobacter pylori and its involvement for antibiotic resistance. Biomed Res Int 2015;2015:914791. doi: 10.1155/2015/914791.

92. Cammarota G, Ianiro G, Bibbò S, et al. Culture-guided treatment approach for Helicobacter pyloriinfection: Review of the literature. World J Gastroenterol. 2014;20(18):520511. doi: 10.3748/wjg.v20.i18.5205.

93. Cammarota G, Branca G, Ardito F, et al. Biofilm demolition and antibiotic treatment to eradicate resistant Helicobacter pylori: a clinical trial. Clin Gastroenterol Hepatol. 2010;8:817-20. doi: 10.1016/j.cgh.2010.05.006.

94. Graham DY, Calvet X. Guide Regarding Choice of Second-line Therapy to Obtain a High Cumulative Cure Rate. Helicobacter. 2012;17:243-5. doi: 10.1111/j.15235378.2012.00952.x

95. Urrego JA, Otero W, Trespalacios A. Avances en la búsqueda de la vacuna contra Helicobacter pylori. MÉD.UIS. 2017;30(3):111-20.

96. Zeng M, Mao XH, Li JX, Tong WD, Wang B, Zhang YJ, et al. Efficacy, safety, and immunogenicity of an oral recombinant Helicobacter pylori vaccine in children in China: a randomised, double-blind, placebo-controlled, phase 3 trial. Lancet. 2015;386(10002):1457-64. doi: 10.1016/S01406736(15)60310-5.

97. Sutton P. At last, vaccine-induced protection against Helicobacter pylori. Lancet. 2015;386(1002):1424-5. doi: 10.1016/S0140-6736(15)60579-7. 